\title{
Modificación de la escala de Alvarado con semiología del dolor para el diagnóstico de apendicitis aguda
}

\author{
Modification of Alvarado score with pain semiology for the diagnosis of acute \\ appendicitis
}

\author{
Ernesto A. Dzib-Calan ${ }^{*}$, Manuel A. Hernández-Rojas", Chiharu Murata², Jorge I. Morales-Pérez", \\ Ivanhoe Larracilla-Salazar ${ }^{1}$ y Gustavo Leal-Mérida ${ }^{3}$ \\ 'Servicio de Cirugía, Hospital General Naval de Alta Especialidad; '2Departamento de Investigación, Instituto Nacional de Pediatría; ${ }^{3}$ Áreas \\ Quirúrgicas, Hospital General Naval de Alta Especialidad. Ciudad de México, México
}

\begin{abstract}
Resumen
Antecedentes: La apendicitis aguda es la patología quirúrgica más frecuente en los servicios de urgencias. Afecta aproximadamente al 9\% de la población. Su diagnóstico diferencial es en extremo difícil, en especial en ancianos, niños y mujeres en edad fértil. La escala de Alvarado (EA) es una herramienta útil para la sospecha diagnóstica de apendicitis. Nuestra experiencia sugiere la posibilidad de mejorar el desempeño diagnóstico de esta escala al incluir la semiología del dolor. Objetivo: Comparar la utilidad diagnóstica de la EA con y sin incluir semiología del dolor utilizada en el Servicio de Cirugía General del Hospital General Naval de Alta Especialidad, así como identificar el menor número de variables útiles para el diagnóstico. Método: Fueron revisados los expedientes de todos los pacientes operados por sospecha de apendicitis aguda de marzo de 2015 a marzo de 2017. Los pacientes incluidos cuentan con expediente completo y reporte histopatológico. Para cada paciente se establecieron el diagnóstico de apendicitis con la aplicación de la EA y agregando la semiología del dolor, y se contrastó el resultado con el diagnóstico histopatológico. Se estimaron los parámetros de utilidad diagnóstica de acuerdo con los puntos de corte determinados por el modelo de regresión logística y las curvas ROC. Resultados: La EA modificada con semiología del dolor mostró una mayor utilidad diagnóstica que la escala original. Se identificaron, además, seis variables con un rendimiento satisfactorio. Conclusión: La semiología del dolor puede mejorar la utilidad diagnóstica de la actual EA.
\end{abstract}

PALABRAS CLAVE: Apendicetomía. Apendicitis aguda. Diagnóstico. Escala de Alvarado.

\begin{abstract}
Background: Acute appendicitis is the most frequent surgical pathology in the emergency services. It affects approximately $9 \%$ of the population. Its differential diagnosis is extremely difficult especially in the elderly, children and women of childbearing age. The Alvarado score (AS) is a useful tool for suspected diagnosis of appendicitis. Our experience suggests the possibility of improving the diagnostic performance of this scale by including pain semiology. Objective: To compare the diagnostic utility of AS with and without including pain semiology used in the general surgery service of the high specialty naval hospital, as well as to identify fewer useful variables for the diagnosis. Method: The files of all the patients operated for the suspicion of acute appendicitis from March 2015 to March of the year 2017 were reviewed. The included patients have complete file and histopathological report. For each patient, diagnosis of appendicitis was established with the application of the AS and another, adding the pain semiology, and the result was contrasted with the histopathological diagnosis. The parameters of diagnostic utility were estimated, based on the cut points determined by the logistic regression model and the ROC curves. Results: AS
\end{abstract}

\author{
Correspondencia: \\ *Ernesto A. Dzib-Calan \\ Avda. Alfredo V. Bonfil, 33-302 \\ Col. Presidentes Ejidales, Primera Sección, Coapa \\ C.P. 04470 , Ciudad de México, México \\ E-mail: eangel_dc@hotmail.com
}

Fecha de recepción: 04-11-2018

Fecha de aceptación: 08-07-2019

DOI: 10.24875/CIRU.19000866
Cir Cir. 2019;87:674-681 www.cirugiaycirujanos.com 
modified with pain semiology showed greater diagnostic utility than the original scale. Six variables were also identified with a satisfactory performance. Conclusion: Pain semiology can improve the diagnostic utility of the current AS.

KEY WORDS: Appendectomy. Acute appendicitis. Diagnosis. Alvarado score.

\section{Introducción}

La apendicitis aguda es la patología quirúrgica más frecuente en los servicios de urgencias. Afecta aproximadamente al $9 \%$ de la población ${ }^{1}$. La letalidad de esta condición es del $0.7 \%{ }^{2}$. Además, el número de apendicetomías negativas permanece en un rango del $10-15 \%$, aunque han disminuido en las décadas recientes debido a las mejores técnicas de diagnóstico ${ }^{3,4}$. Su diagnóstico diferencial es en extremo difícil, especialmente en ancianos, niños y mujeres de edad fértil en quienes puede simular numerosas condiciones ${ }^{4,5}$. El diagnóstico de apendicitis aguda se basa en la anamnesis, la exploración física y estudios de laboratorio e imagen. La exactitud diagnóstica puede ser mejorada con el uso del ultrasonido y la tomografía computarizada. El ultrasonido es fácil de realizar y de bajo costo, pero es dependiente del operador. La tomografía computarizada es de alto costo y expone al paciente a radiación, pero con la ayuda de esta sensible herramienta diagnóstica la frecuencia de apendicetomías negativas ha disminuido de manera significativa ${ }^{6}$. Se han desarrollado diversas escalas diagnósticas para la apendicitis aguda. La escala de Alvarado (EA), introducida en 1986 por el Dr. Alvarado7, es una de las escalas diagnósticas más comúnmente utilizada y se le ha reportado una sensibilidad del $53 \%$ y una especificidad del $88 \%^{8-10}$. Se aplica esta escala en el diagnóstico de los pacientes con sospecha de apendicitis aguda en el servicio de cirugía general del Hospital General Naval de Alta Especialidad; sin embargo, de acuerdo con la experiencia, se averigua también la semiología del dolor (SD) como un apoyo diagnóstico, y consideramos que esta última puede aportar información útil para mejorar el diagnóstico diferencial, a pesar de que no contamos con un estudio formal para sustentar nuestra percepción empírica.

En la actualidad, a pesar de los diversos auxiliares diagnósticos disponibles, sigue existiendo dificultad diagnóstica. En consideración al desafío para los médicos generales, encargados de la evaluación primaria de los pacientes con cuadros de abdomen agudo, refinar los criterios de clasificación que permitan optimizar el diagnóstico clínico de apendicitis aguda es un tema que cuenta con vigencia, en particular en los servicios de atención ambulatoria y de urgencias del primer nivel de atención. El objetivo del presente estudio fue, por lo tanto, comparar la utilidad diagnóstica de la EA y la combinación de EA y SD. En forma secundaria, también exploramos la combinación de los ítems de la EA y las variables de la SD que sugiriera la posibilidad de obtener una nueva escala.

\section{Método}

Se realizó un estudio de la utilidad diagnóstica, retrospectivo y comparativo. Se incluyeron 300 pacientes intervenidos por sospecha de apendicitis aguda durante el periodo de marzo de 2015 a marzo de 2017 en el servicio de cirugía general del Hospital General Naval de Alta Especialidad. Todos los pacientes incluidos cuentan con expediente completo y reporte histopatológico por el servicio de patología. Para cada uno de ellos se estableció el diagnóstico de apendicitis aguda de dos maneras: con la aplicación de los ítems de la EA y agregando los ítems de SD; el resultado se contrastó con el diagnóstico histopatológico.

Los criterios de inclusión fueron pacientes mayores de 15 años que ingresaron al servicio de urgencias con cuadro de dolor abdominal a quienes se realizó apendicetomía como procedimiento de urgencia. Los pacientes que ingresaron sin sospecha de patología apendicular, sin resultado histopatológico y sin datos completos en el expediente fueron excluidos.

La evaluación y la anamnesis de los pacientes las realizaron los residentes del servicio de cirugía general, supervisados por el cirujano adscrito del turno. Los datos incluidos fueron demográficos (edad y sexo), datos de la SD y signos, síntomas y pruebas de laboratorio incluidos en la EA (Tabla 1). La confirmación de la apendicitis aguda como diagnóstico final se obtuvo en el departamento de patología con el análisis histopatológico del apéndice resecado. Los datos fueron registrados en la base de datos elaborada en una hoja de cálculo del programa Excel.

Se determinó la utilidad de prueba diagnóstica de la EA y de la EA + SD, así como la del conjunto de 


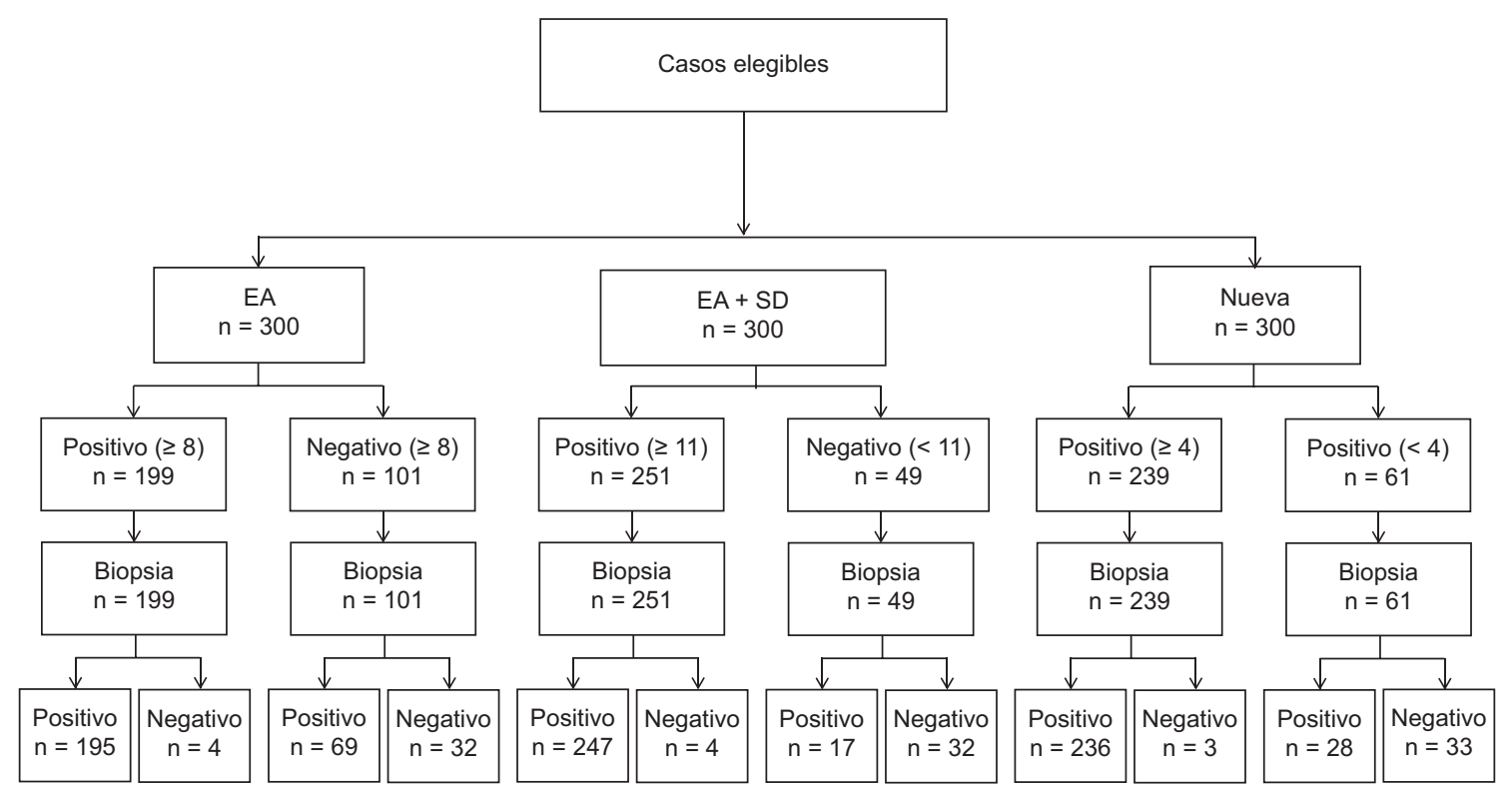

Figura 1. Proceso de inclusión de pacientes y resultados de la escala de Alvarado (EA), la escala de Alvarado más semiología del dolor $(E A+S D)$ y la escala por regresión logística (Nueva), comparados con los diagnósticos histopatológicos.

Tabla 1. Variables que constituyen la escala de Alvarado y variables de semiología del dolor

\begin{tabular}{|c|c|}
\hline Escala de Alvarado & Semiología del dolor \\
\hline Migración del dolor [1] & Inicio progresivo del dolor \\
\hline Anorexia [1] & Evolución $<48 \mathrm{~h}$ \\
\hline Náuseas-vómitos [1] & Localización inicial en el epigastrio \\
\hline Dolor en fosa ilíaca derecha [2] & $\begin{array}{l}\text { Intensidad del dolor en la escala } \\
\text { visual analógica }>7\end{array}$ \\
\hline Rebote positivo [1] & Tipo cólico \\
\hline Fiebre [1] & Agravado con la deambulación \\
\hline Leucocitosis $>10,500 / \mathrm{mm}^{3}$ [2] & Atenuado con la posición en gatillo \\
\hline Neutrofilia $>75 \%[1]$ & Sin recurrencia \\
\hline
\end{tabular}

ítems seleccionados entre las variables estudiadas, por medio de los siguientes parámetros: exactitud global, sensibilidad, especificidad, valor predictivo positivo, valor predictivo negativo, razón de verosimilitud positiva y razón de verosimilitud negativa. La EA, la $\mathrm{EA}+\mathrm{SD}$ y los ítems seleccionados fueron comparados por medio de curva ROC (Receiver Operating Characteristic). Los estimadores puntuales de los parámetros de interés fueron reportados con su intervalo de confianza del $95 \%$. El análisis de los datos se realizó con el paquete estadístico JMP11 (SAS Institute, Inc. NC, USA) y MedCalc (MedCalc Software, Ostend, Belgium).

El presente trabajo se realizó de acuerdo con los principios de la Declaración de Helsinki.

\section{Resultados}

Se incluyeron el estudio los 300 pacientes operados por sospecha de apendicitis aguda (Fig. 1), de los cuales $36(12 \%)$ fueron negativos de acuerdo con el diagnóstico histopatológico. En la distribución de edad no se halló diferencia estadísticamente significativa entre los grupos de pacientes positivos y negativos de acuerdo con el resultado de la biopsia (34.8 \pm 13.2 vs. $39.1 \pm 14.8$ años; $p=0.078$ ). Hubo una proporción de mujeres significativamente mayor en el grupo de negativos (92 vs. $51 \% ; p<0.001$ ).

La mayoría de los pacientes (83.6\%) se presentaron dentro de las primeras 48 horas de la aparición de los síntomas.

La distribución de frecuencia de los datos semiológicos quedó de la siguiente manera: el inicio progresivo del dolor se presentó en 235 pacientes (89\%), la evolución fue menor de 48 horas en 233 pacientes $(88 \%)$, la localización inicial fue en el epigastrio en 223 pacientes (84\%), la intensidad del dolor según la escala visual analógica (EVA) fue $>7$ en 244 pacientes $(92 \%)$, el dolor fue de tipo cólico en 216 pacientes (82\%), agravado con la deambulación en 203 pacientes $(57 \%)$ y atenuado con la posición en gatillo en 195 pacientes $(74 \%)$, y refirieron no haber presentado recurrencia del dolor 230 pacientes (87\%).

En la distribución de frecuencia de los parámetros incluidos en la EA, con respecto a los síntomas clínicos, la migración del dolor a la fosa ilíaca 
Tabla 2. Comparación de las variables demográficas y de las variables que constituyen los ítems de la escala de Alvarado y las variables de la semiología del dolor que se utilizan en el servicio de cirugía del Hospital General Naval de Alta Especialidad

\begin{tabular}{|c|c|c|c|c|c|}
\hline \multirow[t]{2}{*}{ Variables } & \multicolumn{2}{|c|}{ Apendicitis aguda } & \multicolumn{2}{|c|}{ Tamaño de efecto } & \multirow[t]{2}{*}{ p } \\
\hline & Positivo $(n=264)$ & Negativo $(n=36)$ & Valor & Interpretación & \\
\hline Edad [años], media (DE) & $34.8(13.2)$ & $39.1(14.8)$ & 0.32 & Pequeño & 0.078 \\
\hline Sexo [femenino], n (\%) & $134(51 \%)$ & $33(92 \%)$ & 2.33 & Muy grande & $<0.001$ \\
\hline Ítems de Alvarado [suma cruda de + ], media (DE) & $8.4(1.9)$ & $4.7(2.4)$ & 2.02 & Muy grande & $<0.001$ \\
\hline Migración del dolor [+], n (\%) & $243(92 \%)$ & $14(39 \%)$ & 0.49 & Mediano & $<0.001$ \\
\hline Anorexia $[+], n(\%)$ & $203(77 \%)$ & $3(8 \%)$ & 0.48 & Mediano & $<0.001$ \\
\hline Náuseas-vómitos [+], n (\%) & $209(79 \%)$ & $16(44 \%)$ & 0.26 & Pequeño & $<0.001$ \\
\hline Dolor en fosa ilíaca derecha [+], n (\%) & $242(92 \%)$ & $24(67 \%)$ & 0.26 & Pequeño & $<0.001$ \\
\hline Rebote positivo $[+], \mathrm{n}(\%)$ & $244(92 \%)$ & $17(72 \%)$ & 0.44 & Mediano & $<0.001$ \\
\hline Fiebre $[+], n(\%)$ & $150(57 \%)$ & $12(33 \%)$ & 0.15 & Pequeño & 0.008 \\
\hline Leucocitosis > 10,500/mm ${ }^{3}[+], \mathrm{n}(\%)$ & $233(88 \%)$ & $20(56 \%)$ & 0.29 & Pequeño & $<0.001$ \\
\hline Neutrofilia > 75\% [+], n (\%) & $220(83 \%)$ & $20(56 \%)$ & 0.23 & Pequeño & $<0.001$ \\
\hline Semiología del dolor [suma cruda de +], media (DE) & $15.1(2.7)$ & $8.1(3.4)$ & 2.55 & Muy grande & $<0.001$ \\
\hline Inicio progresivo del dolor [+], n (\%) & $235(89 \%)$ & $27(75 \%)$ & 0.14 & Pequeño & 0.018 \\
\hline Evolución < 48 h [+], n (\%) & $233(88 \%)$ & $18(50 \%)$ & 0.34 & Mediano & $<0.001$ \\
\hline Localización inicial en el epigastrio [+], n (\%) & $223(84 \%)$ & $5(14 \%)$ & 0.54 & Grande & $<0.001$ \\
\hline Intensidad del dolor en la escala visual analógica > $7[+], \mathrm{n}(\%)$ & $244(92 \%)$ & $26(72 \%)$ & 0.22 & Pequeño & $<0.001$ \\
\hline Tipo cólico [+], n (\%) & $216(82 \%)$ & $10(28 \%)$ & 0.41 & Mediano & $<0.001$ \\
\hline Agravado con la deambulación [+], n (\%) & $203(57 \%)$ & $16(44 \%)$ & 0.24 & Pequeño & $<0.001$ \\
\hline Atenuado con la posición en gatillo [+], n (\%) & $195(74 \%)$ & $5(14 \%)$ & 0.41 & Mediano & $<0.001$ \\
\hline Sin recurrencia $[+], \mathrm{n}(\%)$ & $230(87 \%)$ & $13(36 \%)$ & 0.41 & Mediano & $<0.001$ \\
\hline
\end{tabular}

derecha se presentó en 243 pacientes (92\%), la anorexia se presentó en 203 pacientes (77\%), y las náuseas y los vómitos en 209 pacientes (79\%). En cuanto a los signos de la exploración física, el dolor en la fosa ilíaca derecha se presentó en 242 pacientes (92\%), el rebote positivo en 244 pacientes (92\%) y la fiebre en 150 pacientes (57\%). En relación con los resultados de la biometría hemática, la

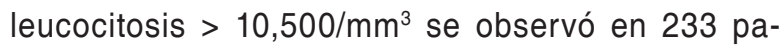
cientes $(88 \%)$ y la neutrofilia $>75 \%$ en 220 pacientes $(83 \%)$.

Tanto en la EA como en la SD, la suma del puntaje presentó una diferencia importante entre los pacientes con resultado positivo y los pacientes con resultado negativo en el estudio histopatológico, siendo en la EA de 8.4 (1.9) vs. 4.7 (2.4), respectivamente, y en la SD de 15.1 (2.7) vs. 8.1 (3.4), respectivamente; en ambos, la diferencia fue muy significativa $(p<0.001)$ y el tamaño de efecto fue muy grande de acuerdo con el criterio de Cohen (EA 2.02 y SD 2.55). Al comparar los ítems de la EA por separado, como era de esperar, todos fueron significativamente diferentes entre los grupos de pacientes positivos y negativos, y con respecto al tamaño de efecto, en «migración del dolor», «anorexia»y «rebote positivo» fue mediano, mientras que en «náusea-vómito», «dolor en fosa ilía-

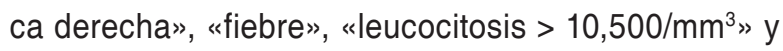
«neutrofilia > 75\%" fue pequeño. Entre las variables de la SD se encontró un tamaño de efecto grande en la variable «localización inicial en epigastrio»; presentaron un tamaño de efecto mediano "evolución < 48 h», «tipo cólico», «atenuado con la posición en gatillo» y «sin recurrencia»; y tuvieron un tamaño de efecto pequeño «inicio progresivo del dolor», «intensidad del dolor en la EVA > 7» y «agravado con la deambulación» (Tabla 2). 
Tabla 3. Modelos de regresión logística para predecir el diagnóstico histopatológico de apendicitis por la escala de Alvarado, la escala de Alvarado más semiología del dolor y la nueva puntuación simplificada

\begin{tabular}{|c|c|c|c|c|c|}
\hline y & $x$ & $\beta(D E)$ & $\chi^{2}$ & $\mathbf{R}^{2}$ & $\mathbf{p}^{*}$ \\
\hline \multirow[t]{3}{*}{ Dx. Apendicitis } & Whole model & & 68.59 & 0.39 & $<0.0001$ \\
\hline & Intercept & $-2.24(0.58)$ & 14.95 & & 0.0001 \\
\hline & Escala de Alvarado & $0.62(0.09)$ & 47.55 & & $<0.0001$ \\
\hline \multirow[t]{3}{*}{ Dx. Apendicitis } & Whole model & & 115.33 & 0.61 & $<0.0001$ \\
\hline & Intercept & $-5.94(1.06)$ & 31.52 & & $<0.0001$ \\
\hline & Alvarado+SEMDOL & $0.68(0.10)$ & 45.97 & & $<0.0001$ \\
\hline \multirow[t]{3}{*}{ Dx. Apendicitis } & Whole model & & 132.54 & 0.69 & $<0.0001$ \\
\hline & Intercept & $-3.70(0.72)$ & 26.43 & & $<0.0001$ \\
\hline & Score simplificado & $1.70(0.25)$ & 46.97 & & $<0.0001$ \\
\hline
\end{tabular}

DE: desviación estándar; Dx: diagnóstico.

Tabla 4. Utilidad diagnóstica de la escala de Alvarado, la escala de Alvarado más semiología del dolor y la nueva puntuación simplificada para predecir el diagnóstico histopatológico

\begin{tabular}{lccc}
\hline & Escala de Alvarado & Alvarado + SEMDOL & Score simplificado \\
\hline Punto de corte & $\geq 8$ & $\geq 11$ & $\geq 4$ \\
Área bajo la curva & $0.89[0.84,0.94]$ & $0.94[0.90,0.99]$ & $0.96[0.93,0.99]$ \\
Exactitud global & $0.76[0.71,0.81]$ & $0.93[0.90,0.96]$ & $0.90[0.86,0.93]$ \\
Sensibilidad & $0.74[0.69,0.79]$ & $0.94[0.91,0.97]$ & $0.89[0.86,0.93]$ \\
Especificidad & $0.89[0.79,0.99]$ & $0.89[0.79,0.99]$ & $0.92[0.83,1.00]$ \\
LR + & $6.65[2.63,16.79]$ & $8.42[3.34,21.22]$ & $10.73[3.63,31.72]$ \\
LR - & $0.29[0.23,0.37]$ & $0.07[0.05,0.12]$ & $0.12[0.08,0.17]$ \\
\hline LR: Likelihood Ratio (Razón de verosimilitud). & &
\end{tabular}

El reporte de histopatología fue considerado como el método de referencia: 264 piezas fueron diagnosticadas como positivas (88\%) para apendicitis aguda y 36 fueron negativas (12\%).

El análisis de regresión logística mostró que el puntaje de la EA presenta la asociación fuerte del tamaño de efecto muy grande con la positividad y la negatividad del resultado de la biopsia (modelo total: $p<0.001 ; R^{2}$ de Nagelkerke $=0.39$ ), pero al agregar los datos de SD el modelo presentó mayor tamaño de efecto, además de que el modelo fue muy altamente significativo (modelo total: $p<0.001 ; R^{2}$ de Nagelkerke $=0.61)$ (Tabla 3).

Se realizó el análisis mediante ROC de ambas escalas de nuestro estudio y se observó que la EA + SD tuvo mayor capacidad de discriminación que la EA sola. La EA mostró un área bajo la curva de 0.89 , con una sensibilidad de 0.74 y una especificidad de 0.89 , estableciendo un punto de corte de 8 . El valor predictivo positivo y el valor predictivo negativo fueron del
97 y el $31 \%$, respectivamente. Por parte de la EA + SD se observó un área bajo la curva hasta 0.94 , con una sensibilidad de 0.94 y una especificidad de 0.89 , con un punto de corte de 11 , y con un valor predictivo positivo del $98 \%$ y un valor predictivo negativo del $65 \%$.

Al aplicar el procedimiento de selección de variables de paso a paso hacia atrás (backward stepwise selection) al mismo modelo de EA + SD se identificaron seis variables como mejores componentes de la nueva escala: «localización inicial en epigastrio», «tipo cólico», «atenuado con la posición en gatillo», «anorexia», «rebote positivo» y «leucocitosis $>10,500 / \mathrm{mm}^{3}$ ». Entre estas variables, las primeras tres fueron de SD y las otras tres fueron de EA. La $\mathrm{R}^{2}$ de Nagelkerke fue de 0.70 (Tabla 4).

Las curvas ROC correspondientes para cada uno de los modelos fueron: $E A \geq 8$, EA + SD $\geq 11$ y nueva $\geq 4$ (Fig. 2). Los parámetros de utilidad diagnóstica de las tres escalas se presentaron en los cuadros anexos 

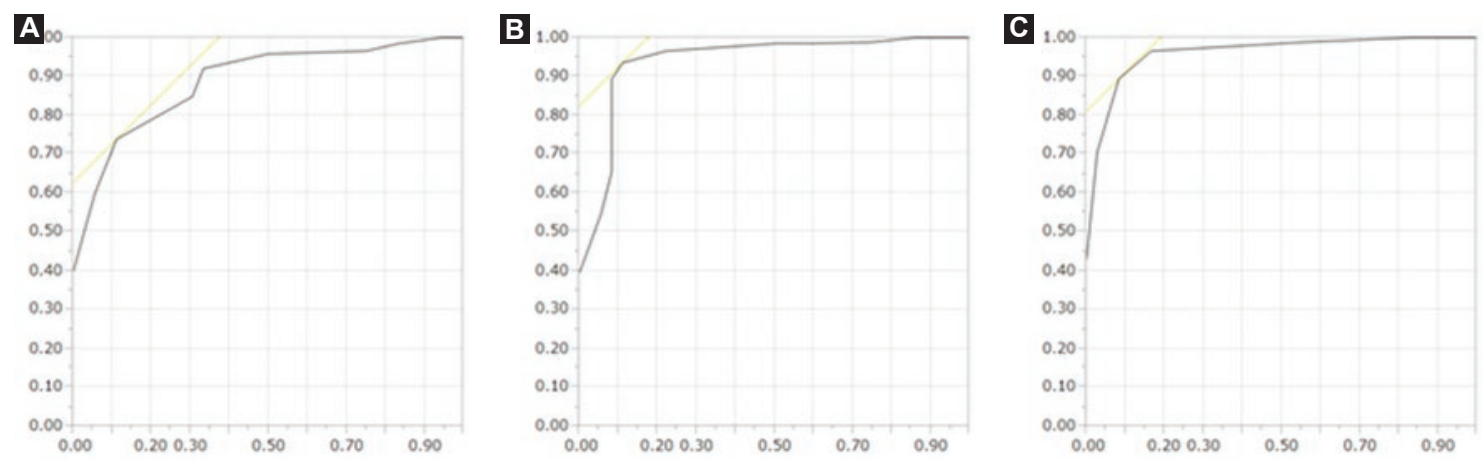

Figura 2. Curvas ROC. A: escala de Alvarado; B: escala de Alvarado más semiología del dolor (EA + SD); C: escala por regresión logística (Nueva). Los puntos de corte y el área bajo curva [intervalo de confianza del 95\%] son $\geq 8$ y 0.89 [0.84,0.94]; $\geq 11$ y 0.94 [0.90,0.99]; y $\geq 4$ y $0.96[0.93,0.99]$, respectivamente.

(Tabla 5). La EA muestra una utilidad suficientemente buena, pero al agregar SD todos los parámetros presentan la mejora, salvo la especificidad, que permaneció sin cambio, y la nueva escala, que consiste en seis variables, supera la utilidad diagnóstica de la EA en todos los parámetros. La razón de verosimilitud específica al estrato de la escala nueva sugiere que este conjunto de seis variables puede ser un modificador útil para la probabilidad posprueba.

\section{Discusión}

En las estadísticas mundiales, la apendicitis aguda figura como la causa más frecuente de abdomen agudo quirúrgico; afecta al $9 \%$ de la población general. La anamnesis y la exploración física continúan siendo la piedra angular en el momento de realizar el diagnóstico. Aunque ningún aspecto de la presentación clínica es específico de la enfermedad, una combinación de signos y síntomas puede orientar el diagnóstico. Es de gran importancia mencionar las complicaciones por la demora en el tratamiento. Dichos cuadros pueden condicionar un mayor tiempo de estancia hospitalaria y en ocasiones reintervenciones quirúrgicas, aumentando la morbimortalidad de los pacientes y generando mayores costos. El tratamiento definitivo es quirúrgico y debe realizarse a la mayor brevedad posible con el fin de evitar complicaciones.

La SD incluye una serie de características de este síntoma que son investigadas de manera obligada por todos los médicos al momento de realizar la evaluación del paciente, y que son de gran importancia y de valor para llegar a un diagnóstico. El dolor abdominal en un cuadro apendicular presenta unas características y una evolución muy particulares, debido a la afección de la propia estructura que genera el dolor visceral inicialmente en el epigastrio y luego localizado
Tabla 5. Stratum Specific Likelihood Ratio de Score simplificado para predecir el diagnóstico histopatológico de apendicitis

\begin{tabular}{lcc}
\hline Estrato & LR + & LR - \\
\hline Score simplificado $\geq 6^{*}$ & $31.42[2.00,494.69]$ & $0.58[0.52,0.65]$ \\
Score simplificado $\geq 5$ & $25.36[3.67,175.47]$ & $0.30[0.25,0.37]$ \\
Score simplificado $\geq 4$ & $10.73[3.63,31.72]$ & $0.12[0.08,0.17]$ \\
Score simplificado $\geq 3$ & $5.79[2.79,12.04]$ & $0.04[0.02,0.08]$ \\
Score simplificado $\geq 2$ & $1.78[1.33,2.38]$ & $0.03[0.01,0.08]$ \\
Score simplificado $\geq 1^{*}$ & $1.21[1.04,1.41]$ & $0.01[<0.00,0.17]$ \\
\hline
\end{tabular}

${ }^{*}$ Con corrección de continuidad de Yates.

LR: Likelihood Ratio (Razón de verosimilitud).

al irritar de manera directa al peritoneo en la región del cuadrante inferior derecho ${ }^{11}$. En el presente estudio, por primera vez, se asigna un valor puntual a esta semiología y se convierte en una escala diagnóstica para una patología específica, demostrando de manera evidente su valor diagnóstico.

En nuestro estudio se tomó como punto de corte para la EA una puntuación de 8, obteniendo como resultado una sensibilidad de 0.74 , una especificidad de 0.89 , un valor predictivo positivo del $97 \%$ y un valor predictivo negativo del $31 \%$. Shuaib, et al. ${ }^{12}$ obtuvieron para la EA modificada una sensibilidad del $82.8 \%$ y una especificidad del $56 \%$, con valor predictivo positivo del $89.3 \%$ y valor predictivo negativo del $42.4 \%$. Singla, et al. ${ }^{13}$ obtuvieron una sensibilidad del $53 \%$ y una especificidad del $100 \%$. Mán, et al. $^{14}$ hallaron una sensibilidad del $69 \%$ y una especificidad del $87 \%$. Sooriakumaran, et al. ${ }^{15}$ reportan una sensibilidad del $50 \%$, una especificidad del $96 \%$, un valor predictivo positivo del $86 \%$ y un valor predictivo negativo del $79 \%$. Kollár, et al..$^{16}$ observaron una sensibilidad del $79 \%$, una especificidad del $76 \%$, un valor predictivo positivo del $65 \%$ y un valor predictivo negativo del $86 \%$. 
Tabla 6. Comparación de la sensibilidad de la escala de Alvarado en diferentes publicaciones

\begin{tabular}{|c|c|c|c|c|}
\hline & \multicolumn{4}{|c|}{ Escala de Alvarado } \\
\hline & Sensibilidad & Especificidad & Valor predictivo positivo & Valor predictivo negativo \\
\hline Sinnet, et al,. $2016^{17}$ & $65 \%$ & $90 \%$ & $96 \%$ & $36 \%$ \\
\hline Golden, et al., $2016^{18}$ & $61 \%$ & $74 \%$ & $53 \%$ & $79 \%$ \\
\hline Özsoy, et al., $2017^{19}$ & $59 \%$ & $83 \%$ & $91 \%$ & $46 \%$ \\
\hline Shuaib, et al., $2017^{12}$ & $83 \%$ & $56 \%$ & $89 \%$ & $42 \%$ \\
\hline Karami, et al., $2017^{20}$ & $78 \%$ & $100 \%$ & $100 \%$ & $38.71 \%$ \\
\hline Díaz-Barrientos, et al., $2018^{21}$ & $75 \%$ & $41.6 \%$ & $93 \%$ & $21.5 \%$ \\
\hline Arroyo-Rangel, et al., $2018^{4}$ & $90 \%$ & $64 \%$ & $94 \%$ & $60 \%$ \\
\hline Este estudio (Alvarado) & $74 \%$ & $89 \%$ & $97 \%$ & $31 \%$ \\
\hline Este estudio (Alvarado+semiología dolor) & $94 \%$ & $89 \%$ & $98 \%$ & $65 \%$ \\
\hline
\end{tabular}

Con respecto a nuestra escala modificada, obtuvimos puntaje de sensibilidad de 0.94 , una especificidad de 0.89 , un valor predictivo positivo del $98 \%$ y un valor predictivo negativo del $65 \%$, observando una mejoría en las puntuaciones generales en comparación con los resultados obtenidos con la EA.

Se realizó el análisis ROC para buscar puntajes de corte en ambos sistemas de puntuación. La EA resultó con un punto de corte de 8 , con un área bajo la curva (AUC) de 0.89. Singla, et al. ${ }^{13}$ reportaron un punto de corte de 4.5 con la EA modificada para las máximas sensibilidad y especificidad, mucho menor que el puntaje original de 7 AUC de 0.580 . Mán, et al. ${ }^{14}$ reportaron en su estudio un punto de corte de 7 para la EA, con AUC de 0.749 . Kollár, et al. ${ }^{16}$ encontraron, con un punto de corte de 7, un AUC de 0.863 para la EA. Se extrajeron los resultados de sensibilidad y especificidad, así como los valores predictivos positivo y negativo de diferentes autores en los últimos años ${ }^{4,12,17-21}$ y se compararon con los resultados encontrados en nuestro estudio (Tabla 6).

Hemos obtenido resultados que muestran la posibilidad de mejorar la utilidad diagnóstica de la EA por integrar las variables de SD. Nuestros hallazgos sustentan la percepción empírica que hemos tenido en nuestro servicio de cirugía general del Hospital General Naval de Alta Especialidad. Además, hemos obtenido un conjunto de seis variables tomados de la EA y de la SD, el cual presentó un rendimiento superior a los dos anteriores.

\section{Conclusión}

La EA puede ser mejorada por integrar SD, y hay seis variables que potencialmente pueden conformar un nuevo instrumento de diagnóstico diferencial. Es necesario realizar un estudio prospectivo, con una mayor muestra y con diferentes poblaciones y condiciones geográficas.

\section{Agradecimientos}

A nuestro asesor metodológico, por enseñarnos la luz donde todos los demás veían oscuridad. Al departamento de cirugía general del Hospital General Naval de Alta Especialidad, por el apoyo brindado.

\section{Conflicto de intereses}

Los autores declaran no tener ningún conflicto de intereses.

\section{Responsabilidades éticas}

Protección de personas y animales. Los autores declaran que para esta investigación no se han realizado experimentos en seres humanos ni en animales.

Confidencialidad de los datos. Los autores declaran que han seguido los protocolos de su centro de trabajo sobre la publicación de datos de pacientes.

Derecho a la privacidad y consentimiento informado. Los autores declaran que en este artículo no aparecen datos de pacientes.

\section{Bibliografía}

1. Anderson JE, Bickler SW, Chang DC, Talamini MA. Examining a common disease with unknown etiology: trends in epidemiology and surgical management of appendicitis in California, 1995-2009. World J Surg. 2012;36:2787-94. 
2. Antal A. [Changes in epidemiology, etiology, diagnostics, and therapy of acute appendicitis]. Orv Hetil. 2009;150:443-6.

3. Raja AS, Wright C, Sodickson AD, Zane RD, Schiff GD, Hanson R, et al. Negative appendectomy rate in the era of CT (an 18-year perspective). Radiology. 2010;256:460-5.

4. Arroyo-Rangel C, Limón IO, Vera AG, Guardiola PM, Sánchez-Valdivieso EA. Sensibilidad, especificidad y fiabilidad de la escala RIPASA en el diagnóstico de apendicitis aguda en relación con la escala de Alvarado. Cir Esp. 2018.

5. Gilmore OJ, Browett JP, Griffin PH, Ross IK, Brodribb AJ, Cooke TJ, et al. Appendicitis and mimicking conditions. A prospective study. Lancet. 1975;2:421-4.

6. Ohle R, O'Reilly F, O'Brien KK, Fahey T, Dimitrov BD. The Alvarado score, for predicting acute appendicitis: a systematic review. BMC Med. 2011:9:139

7. Baidya N, Rodrigues G, Rao A, Khan S. Evaluation of Alvarado score in acute appendicitis: a prospective study. The Internet Journal of Surgery. 2006;9(1).

8. Alvarado A. A practical score for the early diagnosis of acute appendicitis. Ann Emerg Med. 1986;15:557e64.

9. Kalan M, Talbot D, Cunliffe WJ, Rich AJ. Evaluation of the modified AIvarado score in the diagnosis of acute appendicitis: a prospective study. Ann R Coll Surg Engl. 1994;76:418-9.

10. Chong CF, Adi MI, Thien A, Suyoi A, Mackie AJ, Tin AS. Development of the RIPASA score: a new appendicitis scoring system for the diagnosis of acute appendicitis. Singapore Med J. 2010;51:220-5

11. Mayo Ossorio MA, Pacheco García JM, Vázquez Gallego JM. Abdomen agudo. Medicine. 2016;12:363-79.

12. Shuaib A, Shuaib A, Fakhra Z, Marafi B, Alsharaf K, Behbehani A. Evaluation of modified Alvarado scoring system and RIPASA scoring system as diagnostic tools of acute appendicitis. World J Emerg Med. 2017;8:276-80.
13. Singla A, Singla S, Singh M, Singla D. A comparison between modified Alvarado score and RIPASA score in the diagnosis of acute appendicitis. Updates Surg. 2016;68:351-5.

14. Mán E, Simonka Z, Varga A, Rárosi F, Lázár G. Impact of the Alvarado score on the diagnosis of acute appendicitis: comparing clinical judgment, Alvarado score, and a new modified score in suspected appendicitis: a prospective, randomized clinical trial. Surg Endosc. 2014; 28:2398-405.

15. Sooriakumaran $P$, Lovell $D$, Brown R. A comparison of clinical judgment vs the modified Alvarado score in acute appendicitis. Int J Surg. 2005;3:49-52.

16. Kollár D, McCartan DP, Bourke M, Cross KS, Dowdall J. Predicting acute appendicitis? A comparison of the Alvarado Score, the Appendicitis Inflammatory Response Score and clinical assessment. World J Surg. 2015;39:104-9.

17. Sinnet PR, Chellappa PM, Kumar S, Ethirajulu R, Thambi S. Comparative study on the diagnostic accuracy of the RIPASA score over Alvarado score in the diagnosis of acute appendicitis. J Evid Based Med Healthc. $2016 ; 3: 4318-21$

18. Golden SK, Harringa JB, Pickhardt PJ, Ebinger A, Svenson JE, Zhao YQ, et al. Prospective evaluation of the ability of clinical scoring systems and physician-determined likelihood of appendicitis to obviate the need for computed tomography. Emerg Med J. 2016;33:458-64.

19. Ozsoy Z, Yenidoğan E. Evaluation of the Alvarado scoring system in the management of acute appendicitis. Turk J Surg. 2017;33:200-4.

20. Karami MY, Niakan H, Zadebagheri N, Mardani P, Shayan Z, Deilami I. Which one is better? Comparison of the acute inflammatory response, Raja Isteri Pengiran Anak Saleha Appendicitis and Alvarado scoring systems. Ann Coloproctol. 2017;33:227-31.

21. Díaz-Barrientos $C Z$, Aquino-González A, Heredia-Montaño M, Navarro-Tovar F, Pineda-Espinosa MA, Espinosa de Santillana IA. Escala RIPASA para el diagnóstico de apendicitis aguda: comparación con la escala de Alvarado modificada. Rev Gastroenterol Mex. 2018;83:112-6. 